\title{
Betti numbers in multidimensional persistent homology are stable functions
}

\author{
Andrea Cerri Barbara Di Fabio Massimo Ferri \\ Patrizio Frosini Claudia Landi
}

\begin{abstract}
Multidimensional persistence mostly studies topological features of shapes by analyzing the lower level sets of vector-valued functions, called filtering functions. As is well known, in the case of scalar-valued filtering functions, persistent homology groups can be studied through their persistent Betti numbers, i.e. the dimensions of the images of the homomorphisms induced by the inclusions of lower level sets into each other. Whenever such inclusions exist for lower level sets of vector-valued filtering functions, we can consider the multidimensional analogue of persistent Betti numbers. Varying the lower level sets, we get that persistent Betti numbers can be seen as functions taking pairs of vectors to the set of non-negative integers. In this paper we prove stability of multidimensional persistent Betti numbers. More precisely, we prove that small changes of the vector-valued filtering functions imply only small changes of persistent Betti numbers functions. This result can be obtained by assuming the filtering functions to be just continuous. Multidimensional stability opens the way to a stable shape comparison methodology based on multidimensional persistence. In order to obtain our stability theorem, some other new results are proved for continuous filtering functions. They concern the finiteness of persistent Betti numbers for vector-valued filtering functions, and the representation via persistence diagrams of persistent Betti numbers, as well as their stability, in the case of scalarvalued filtering functions. Finally, from the stability of multidimensional persistent Betti numbers we obtain a lower bound for the natural pseudo-distance.
\end{abstract}

Keywords. Multidimensional persistence, persistence diagram, Čech homology, foliation, continuous filtering function, natural pseudo-distance

A. Cerri, B. Di Fabio, M. Ferri, P. Frosini: ARCES and Dipartimento di Matematica, Università di Bologna, Italia; e-mail: \{cerri,difabio,ferri,frosini\}@dm.unibo.it,

C. Landi: Dipartimento di Scienze e Metodi dell'Ingegneria, Università di Modena e Reggio Emilia, Italia; e-mail: clandi@unimore.it

Mathematics Subject Classification (2010): Primary 55N35; Secondary 55N05, $68 \mathrm{U} 05$. 


\section{Introduction}

The study of the topology of data is attracting more and more attention from the mathematical community. This challenging subject of research is motivated by the large amount of scientific contexts where it is required to deal with qualitative geometric information. Indeed, the topological approach allows us to greatly reduce the complexity of the data by focusing the analysis just on their relevant part. This research area is widely discussed in $[1,2]$.

\section{Persistence}

Persistent homology has turned out to be a key mathematical method for studying the topology of data, with applications in an increasing number of fields, ranging from shape description (e.g., [3, 4, 5, 6]) to data simplification [7] and hole detection in sensor networks [8]. Recent surveys on the topic include [9, 10, 11, 12]. Persistent homology describes topological events occurring through the filtration of a topological space $X$. Filtrations are usually expressed by real functions $\varphi: X \rightarrow \mathbb{R}$ called filtering functions. The main idea underlying this approach is that the most important piece of information enclosed in geometrical data is usually the one that is "persistent" with respect to the defining parameters.

The analysis of persistent topological events in the lower level sets of the functions (e.g., creation, merging, cancellation of connected components, tunnels, voids) is important for capturing a global description of the data under study. These events can be encoded in the form of a parameterized version of the Betti numbers known in the literature as persistent Betti numbers [7], a rank invariant [13], and, for the 0th homology, a size function $[14,15,6]$.

\section{Motivations}

Until recently, research on persistence has mainly focused on the use of scalar functions for describing filtrations. The extent to which this theory can be generalized to a situation in which two or more functions characterize the data is currently under investigation $[16,17,18,13]$. This generalization to vector-valued functions is usually known as the Multidimensional Persistence Theory, where the adjective multidimensional refers to the fact that filtering functions are vector-valued, and has no connections with the dimensionality of the space under study. The use of vector-valued filtering functions in this context, introduced in [19] for persistence of homotopy groups, enables the analysis of richer data structures.

One of the most important open questions in current research about multidimensional persistent homology concerns the stability problem. In plain words, we need to determine 
how the computation of invariants in this theory is affected by the unavoidable presence of noise and approximation errors. Indeed, it is clear that any data acquisition is subject to perturbations and, if persistent homology were not stable, then distinct computational investigations of the same object could produce completely different results. Obviously, this would make it impossible to use such a mathematical theory in real applications.

\section{Prior works}

The problem of stability in persistent homology has been studied by Cohen-Steiner, Edelsbrunner and Harer in [20] for scalar filtering functions. By using a descriptor called a persistence diagram, they prove that persistent Betti numbers are stable under perturbations of filtering functions with respect to the max-norm, provided that the considered filtering functions are tame. The same problem is studied in [21] for tame Lipschitz functions. In [22], Chazal et al. introduce the concept of persistence module and prove stability under the assumption that it is finite-dimensional. The problem of stability for scalar filtering functions is also approached in [23], where it is solved by assuming that the considered filtering functions are no more than continuous, but only for the 0th homology.

Multidimensional persistence was firstly investigated in [19] as regards homotopy groups, and by Carlsson and Zomorodian in [24] as regards homology modules. In this context, the first stability result has been obtained for the 0th homology in [16]: A distance between the 0th persistent Betti numbers, also called size functions, has been introduced and proven to be stable under perturbations of continuous vector-valued filtering functions. Such a result has been partially extended in [17] for all homology degrees, under the restrictive assumption that the vector-valued filtering functions are max-tame. This condition is quite technical and, in general, it is unknown when it is satisfied.

\section{Contributions of the paper}

In this paper we present new stability results, for both scalar and vector-valued continuous filtering functions, that are not limited by the restrictions of tameness and max-tameness (see Theorem 3.13 and Theorem 4.4). More precisely, we prove that the persistent Betti numbers of nearby scalar or vector-valued filtering functions are "close to each other" in the sense expressed by a suitable matching distance. Along the way, we also prove the following new theorems.

The first relevant result is the proof of the Finiteness Theorem 2.3, stating that, if the space under study is triangulable and the vector-valued filtering function is continuous, then the associated persistent Betti numbers (hereafter PBNs) are finite. The importance of this result relies on the fact that the finiteness of PBNs is required in a number of intermediate steps eventually leading to the definition of persistence diagrams (Defini- 
tion 3.5). The finiteness of PBNs has revealed to be fundamental also in other related works $[22,20]$. In these papers, it is gained as a direct consequence of assuming that the filtering functions (in [20]) or the persistence modules (in [22]) are tame. On the other hand, the finiteness of PBNs for filtering functions that are just continuous requires a specific proof.

Persistence diagrams are a key ingredient of the present paper together with the use of Čech homology over a field. Indeed, our second relevant result is the Representation Theorem 3.11, stating that, with the use of Čech homology, the PBNs of a scalar-valued filtering function can be completely described by a persistence diagram.

As an immediate consequence of the Representation Theorem 3.11, it follows that any distance between persistence diagrams induces a distance between one-dimensional PBNs. This justifies the introduction of the matching distance, recalled in Definition 3.12, leading us to prove the third relevant result of this paper, the One-Dimensional Stability Theorem 3.13. Roughly speaking, this theorem states that small changes of the considered scalar-valued filtering functions, with respect to the max-norm, induce only small changes in the associated PBNs, with respect to the matching distance.

The One-Dimensional Stability Theorem 3.13 is a necessary step towards the last and main contribution of the paper, i.e. the Multidimensional Stability Theorem 4.4. This stability result for the multidimensional setting requires us to use some ideas recently developed to investigate Multidimensional Size Theory [16]. The proof of multidimensional stability is obtained by reduction to the one-dimensional case, via an appropriate foliation in half-planes of the domain of the PBNs associated with a vector-valued filtering function. Indeed, it is possible to prove that each restriction of a multidimensional PBNs function to one of these half-planes turns out to be a one-dimensional PBNs function of a suitable, possibly non-tame, continuous scalar-valued filtering function (see Theorem 4.2). This approach implies that the comparison of two multidimensional PBNs can be performed leaf by leaf by appropriately measuring the distance of one-dimensional PBNs. Therefore, the stability of multidimensional persistence is a consequence of the one-dimensional persistence stability for continuous filtering functions, i.e. of the OneDimensional Stability Theorem 3.13.

As a corollary of the Multidimensional Stability Theorem 4.4, we obtain a lower bound for the natural pseudo-distance. The natural-pseudo distance, introduced in [25] and further studied in [26, 27], is a dissimilarity measure for topological spaces endowed with vector-valued continuous functions that is intrinsically hard to compute, motivating the interest in methods for its estimation.

\section{Working assumptions}

Following [20], we will work with triangulable topological spaces. 
The considered filtering functions are assumed to be just continuous, thus weakening the tameness and the max-tameness requirements of [20] and [17], respectively. The reasons of this choice are manifold. Firstly, our generalization from tame to continuous functions is a positive answer to a question risen in [20]. Secondly, the one-dimensional reduction of multidimensional persistent homology is not possible in the setting of tame functions, as it was already observed in [17], but it luckily does in the wider setting of continuous functions.

The choice of working with Čech homology is motivated by the fact that, having the continuity axiom, it allows us to prove the Representation Theorem 3.11. Even assuming tameness, this result would not hold for singular and simplicial theories, which guarantee a complete description of one-dimensional PBNs only outside a set of vanishing measure, as explained in Section 2.2.1. In the framework of persistence, Čech homology has already been considered by Robins in [28, 29]. Moreover, the Čech approach to homology theory is currently being investigated for computational purposes [30].

To conclude, we find it necessary to warn the reader of a technical decision we have taken. This paper arises from the observation that the proof line already adopted for 0th homology perfectly works also for $k$ th homology once some basic properties are granted, with suitable technical adaptations. In the following, we shall state and prove completely these necessary properties but we will only sketch whatever can be found in the original papers for the sake of conciseness, fairness and readability. However, in the present paper, terminology will stick to that of Persistence Theory as much as possible.

\section{PBNs: Definitions and first properties}

In this paper, the following relations $\preceq$ and $\prec$ are defined in $\mathbb{R}^{n}$ : for $\vec{u}=\left(u_{1}, \ldots, u_{n}\right)$ and $\vec{v}=\left(v_{1}, \ldots, v_{n}\right)$, we say $\vec{u} \preceq \vec{v}$ (resp. $\vec{u} \prec \vec{v}$ ) if and only if $u_{i} \leq v_{i}$ (resp. $u_{i}<$ $v_{i}$ ) for every index $i=1, \ldots, n$. Moreover, $\mathbb{R}^{n}$ is endowed with the usual max-norm: $\left\|\left(u_{1}, u_{2}, \ldots, u_{n}\right)\right\|_{\infty}=\max _{1 \leq i \leq n}\left|u_{i}\right|$.

We shall use the following notations: $\Delta^{+}$will be the open set $\left\{(\vec{u}, \vec{v}) \in \mathbb{R}^{n} \times \mathbb{R}^{n}\right.$ : $\vec{u} \prec \vec{v}\}$. For every $n$-tuple $\vec{u}=\left(u_{1}, \ldots, u_{n}\right) \in \mathbb{R}^{n}$ and for every function $\vec{\varphi}: X \rightarrow \mathbb{R}^{n}$, we shall denote by $X\langle\vec{\varphi} \preceq \vec{u}\rangle$ the set $\left\{x \in X: \varphi_{i}(x) \leq u_{i}, i=1, \ldots, n\right\}$.

The definition below extends the concept of the persistent homology group to a multidimensional setting.

Definition 2.1 (Persistent homology group). Let $k \in \mathbb{Z}$. Let $X$ be a topological space, and $\vec{\varphi}: X \rightarrow \mathbb{R}^{n}$ a continuous function. Let $\pi_{k}^{(\vec{u}, \vec{v})}: \check{H}_{k}(X\langle\vec{\varphi} \preceq \vec{u}\rangle) \rightarrow \check{H}_{k}(X\langle\vec{\varphi} \preceq \vec{v}\rangle)$ be the homomorphism induced by the inclusion map $\pi^{(\vec{u}, \vec{v})}: X\langle\vec{\varphi} \preceq \vec{u}\rangle \hookrightarrow X\langle\vec{\varphi} \preceq \vec{v}\rangle$ with $\vec{u} \preceq \vec{v}$, where $\check{H}_{k}$ denotes the $k$ th $\check{C}$ ech homology group. If $\vec{u} \prec \vec{v}$, the image of $\pi_{k}^{(\vec{u}, \vec{v})}$ 
is called the multidimensional kth persistent homology group of $(X, \vec{\varphi})$ at $(\vec{u}, \vec{v})$, and is denoted by $\check{H}_{k}^{(\vec{u}, \vec{v})}(X, \vec{\varphi})$.

In other words, the group $\check{H}_{k}^{(\vec{u}, \vec{v})}(X, \vec{\varphi})$ contains all and only the homology classes of cycles born before or at $\vec{u}$ and still alive at $\vec{v}$.

For details about Čech homology, the reader can refer to [31, Ch. IX].

In what follows, we shall work with coefficients in a field $\mathbb{K}$, so that homology groups are vector spaces. Therefore, they can be completely described by their dimension, leading to the following definition (cf. $[13,7])$.

Definition 2.2 (Persistent Betti Numbers Function). The function $\beta_{\vec{\varphi}}: \Delta^{+} \rightarrow \mathbb{N} \cup\{\infty\}$ defined by

$$
\beta_{\vec{\varphi}}(\vec{u}, \vec{v})=\operatorname{dimim} \pi_{k}^{(\vec{u}, \vec{v})}=\operatorname{dim} \check{H}_{k}^{(\vec{u}, \vec{v})}(X, \vec{\varphi})
$$

will be called the persistent Betti numbers function of $\vec{\varphi}$, briefly PBNs.

Obviously, for each $k \in \mathbb{Z}$, we have different PBNs $\beta_{\vec{\varphi}}$ of $\vec{\varphi}$ (which should be denoted $\beta_{\vec{\varphi}, k}$, say) but, for the sake of notational simplicity, we omit adding any reference to $k$. This will also apply to the notations used for other concepts in this paper, such as multiplicities and persistence diagrams.

We shall prove in Theorem 2.3 that, if $X$ is triangulable, $\beta_{\vec{\varphi}}$ never attains the value $\infty$.

\subsection{Properties of multidimensional PBNs}

The next Theorem 2.3 ensures that, if the considered topological spaces are assumed to be triangulable, multidimensional PBNs (Definition 2.2) are finite even dropping the tameness condition requested in [20]. We underline that the PBNs finiteness is not obvious from the assumption that the space is triangulable. Indeed, the lower level sets with respect to a continuous function are not necessarily triangulable spaces.

Theorem 2.3 (Finiteness). Let $X$ be a triangulable space, and $\vec{\varphi}: X \rightarrow \mathbb{R}^{n}$ a continuous function. Then, for every $(\vec{u}, \vec{v}) \in \Delta^{+}$, it holds that $\beta_{\vec{\varphi}}(\vec{u}, \vec{v})<+\infty$.

Proof. Since $X$ is triangulable, we can assume that it is the support of a simplicial complex $K$ and that a distance $d$ is defined on $X$, compatible with its topology.

Let us fix $(\vec{u}, \vec{v}) \in \Delta^{+}$, and choose a real number $\varepsilon>0$ such that, setting $\vec{\varepsilon}=$ $(\varepsilon, \ldots, \varepsilon) \in \mathbb{R}^{n}, \vec{u}+2 \vec{\varepsilon} \prec \vec{v}$.

We now show that there exist a function $\vec{\psi}: X \rightarrow \mathbb{R}^{n}$, a subdivision $K^{\prime \prime}$ of $K$, and a triangulation $L^{\prime \prime}$ of $\vec{\psi}(X)$, such that $(i)$ the triple $\left(\vec{\psi}, K^{\prime \prime}, L^{\prime \prime}\right)$ is simplicial, and $(i i)$ $\max _{x \in X}\|\vec{\varphi}(x)-\vec{\psi}(x)\|_{\infty}<\varepsilon$.

Indeed, by the uniform continuity of each component $\varphi_{i}$ of $\vec{\varphi}$, there exists a real number $\delta>0$ such that, for $i=1, \ldots, n,\left|\varphi_{i}(x)-\varphi_{i}\left(x^{\prime}\right)\right|<\varepsilon$ for every $x, x^{\prime} \in X$ with 
$d\left(x, x^{\prime}\right)<\delta$. We take a subdivision $K^{\prime}$ of $K$ such that mesh $\left(K^{\prime}\right)<\delta$, and define $\vec{\psi}(x)=\vec{\varphi}(x)$ for every vertex $x$ of $K^{\prime}$. Next, we consider the linear extension of $\vec{\psi}$ to the other simplices of $K^{\prime}$. In this way, $\vec{\psi}$ is linear on each simplex of $K^{\prime}$.

Since $\vec{\psi}$ is piecewise linear, $\vec{\psi}(X)$ is the underlying space of a simplicial complex $L^{\prime}$. By taking suitable subdivisions $K^{\prime \prime}$ of $K^{\prime}$ and $L^{\prime \prime}$ of $L^{\prime}, \vec{\psi}$ also maps simplices into simplices and therefore $\left(\vec{\psi}, K^{\prime \prime}, L^{\prime \prime}\right)$ is simplicial (cf. [32, Thm. 2.14]). This proves $(i)$.

To see $(i i)$, let us consider a point $x$ belonging to a simplex in $K^{\prime}$, of vertices $v_{1}, \ldots, v_{r}$. Since $x=\sum_{i=1}^{r} \lambda_{i} \cdot v_{i}$, with $\lambda_{1}, \ldots, \lambda_{r} \geq 0$ and $\sum_{i=1}^{r} \lambda_{i}=1$, and $\vec{\psi}$ is linear on each simplex, it follows that $\|\vec{\varphi}(x)-\vec{\psi}(x)\|_{\infty}=\left\|\vec{\varphi}(x)-\sum_{i=1}^{r} \lambda_{i} \cdot \vec{\psi}\left(v_{i}\right)\right\|_{\infty}=\| \vec{\varphi}(x)-$ $\sum_{i=1}^{r} \lambda_{i} \cdot \vec{\varphi}\left(v_{i}\right)\left\|_{\infty}=\right\| \sum_{i=1}^{r} \lambda_{i} \cdot \vec{\varphi}(x)-\sum_{i=1}^{r} \lambda_{i} \cdot \vec{\varphi}\left(v_{i}\right)\left\|_{\infty} \leq \sum_{i=1}^{r} \lambda_{i}\right\| \vec{\varphi}(x)-\vec{\varphi}\left(v_{i}\right) \|_{\infty}<$ $\varepsilon$.

We now prove that, since $\left(\vec{\psi}, K^{\prime \prime}, L^{\prime \prime}\right)$ is simplicial, it holds that $\check{H}_{k}(X\langle\vec{\psi} \preceq \vec{u}+\vec{\varepsilon}\rangle)$ is finitely generated. Indeed, since the intersection between a simplex and a half-space is triangulable, there exists a subdivision $L^{\prime \prime \prime}$ of $L^{\prime \prime}$ such that $\vec{\psi}(X) \cap\left\{\vec{x} \in \mathbb{R}^{n}: \vec{x} \preceq \vec{u}+\vec{\varepsilon}\right\}$ is triangulated by a subcomplex of $L^{\prime \prime \prime}$. By [32, Lemma 2.16], there is a subdivision $K^{\prime \prime \prime}$ of $K^{\prime \prime}$ such that $\left(\vec{\psi}, K^{\prime \prime \prime}, L^{\prime \prime \prime}\right)$ is simplicial. It follows that $X\langle\vec{\psi} \preceq \vec{u}+\vec{\varepsilon}\rangle$ is triangulable, and hence $\check{H}_{k}(X\langle\vec{\psi} \preceq \vec{u}+\vec{\varepsilon}\rangle)$ is finitely generated.

Since $\vec{u}+2 \vec{\varepsilon} \prec \vec{v}$ and $\max _{x \in X}\|\vec{\varphi}(x)-\vec{\psi}(x)\|_{\infty}<\varepsilon$, we have the inclusions $X\langle\vec{\varphi} \preceq$ $\vec{u}\rangle \stackrel{i}{\rightarrow} X\langle\vec{\psi} \preceq \vec{u}+\vec{\varepsilon}\rangle \stackrel{j}{\rightarrow} X\langle\vec{\varphi} \preceq \vec{v}\rangle$, inducing the homomorphisms $\check{H}_{k}(X\langle\vec{\varphi} \preceq \vec{u}\rangle) \stackrel{i_{k}}{\rightarrow}$ $\check{H}_{k}(X\langle\vec{\psi} \preceq \vec{u}+\vec{\varepsilon}\rangle) \stackrel{j_{k}}{\rightarrow} \check{H}_{k}(X\langle\vec{\varphi} \preceq \vec{v}\rangle)$. By recalling that $\check{H}_{k}(X\langle\vec{\psi} \preceq \vec{u}+\vec{\varepsilon}\rangle)$ is finitely generated, and since $\operatorname{dim} \operatorname{im} j_{k} \circ i_{k} \leq \operatorname{dimim} j_{k}$, we obtain the claim.

We point out that, in our setting, the finiteness of PBNs would not be guaranteed if they were defined also on the boundary of $\Delta^{+}$, i.e. for pairs $(\vec{u}, \vec{v})$ such that not all coordinates differ. This motivates our choice of working only on $\Delta^{+}$.

The following Lemmas 2.4 and 2.5 generalize to the multidimensional setting analogous results valid for $n=1$. We omit the trivial proof of Lemma 2.4.

Lemma 2.4 (Monotonicity). $\beta_{\vec{\varphi}}(\vec{u}, \vec{v})$ is non-decreasing in $\vec{u}$ and non-increasing in $\vec{v}$ (with respect to the partial order relation $\preceq$ ).

Lemma 2.5 (Diagonal Jump). Let $X, Y$ be two homeomorphic triangulable spaces, and $f: X \rightarrow Y$ a homeomorphism. Let $\vec{\varphi}: X \rightarrow \mathbb{R}^{n}, \vec{\psi}: Y \rightarrow \mathbb{R}^{n}$ be continuous functions. Then, for every $(\vec{u}, \vec{v}) \in \Delta^{+}$, and for every $h \in \mathbb{R}$ such that $\max _{x \in X}\|\vec{\varphi}(x)-\vec{\psi} \circ f(x)\|_{\infty} \leq$ $h$, setting $\vec{h}=(h, \ldots, h) \in \mathbb{R}^{n}$, we have $\beta_{\vec{\varphi}}(\vec{u}-\vec{h}, \vec{v}+\vec{h}) \leq \beta_{\vec{\psi}}(\vec{u}, \vec{v})$.

Proof. Since $\max _{x \in X}\|\vec{\varphi}(x)-\vec{\psi} \circ f(x)\|_{\infty} \leq h$, we have the following commutative diagram 


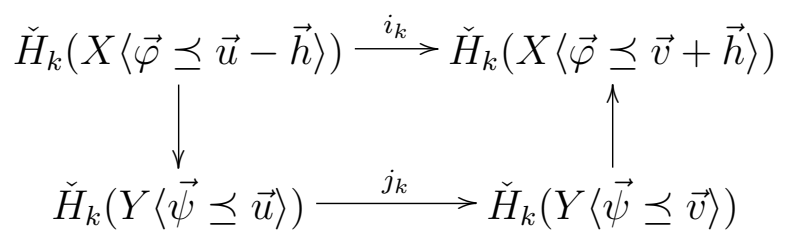

where $i_{k}$ and $j_{k}$ are induced by inclusions, and the vertical homomorphisms are induced by restrictions of $f$ and $f^{-1}$, respectively. Thus the commutativity of the diagram implies that $\operatorname{dim} \operatorname{im} i_{k} \leq \operatorname{dimim} j_{k}$, yielding the claim.

\subsection{Properties of one-dimensional PBNs}

Now we confine ourselves to the case $n=1$. Therefore, for the sake of simplicity, the symbols $\vec{\varphi}, \vec{u}, \vec{v}$ will be replaced by $\varphi, u, v$, respectively. We remark that $\Delta^{+}$reduces to be the set $\left\{(u, v) \in \mathbb{R}^{2}: u<v\right\}$. Moreover, we use the following notations: $\Delta=\partial \Delta^{+}$, $\Delta^{*}=\Delta^{+} \cup\{(u, \infty): u \in \mathbb{R}\}$, and $\bar{\Delta}^{*}=\Delta^{*} \cup \Delta$.

\subsubsection{Right-continuity of one-dimensional PBNs}

In what follows we shall prove that, using Čech homology, the one-dimensional PBNs function is right-continuous with respect to both $u$ and $v$, i.e. $\lim _{u \rightarrow \bar{u}^{+}} \beta_{\varphi}(u, v)=\beta_{\varphi}(\bar{u}, v)$ and $\lim _{v \rightarrow \bar{v}^{+}} \beta_{\varphi}(u, v)=\beta_{\varphi}(u, \bar{v})$. This property will be necessary to completely characterize PBNs by a persistence diagram, a descriptor whose definition will be recalled later in this section. In the absence of right-continuity, persistence diagrams describe PBNs only almost everywhere, thus justifying the use of Čech homology in this context.

The next example shows that the right-continuity in the variable $u$ does not always hold when persistent homology groups are defined using simplicial or singular homology, even under the tameness assumption.

Example 2.6. Let $X$ be a closed rectangle of $\mathbb{R}^{2}$ containing a Warsaw circle (see Figure 1). Let also $\varphi: X \rightarrow \mathbb{R}$ be the Euclidean distance from the Warsaw circle.

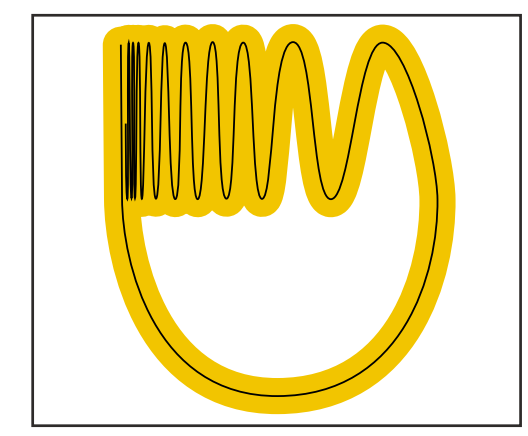

Figure 1: A lower level set $X\langle\varphi \leq u\rangle$, for a sufficiently small $u>0$, as considered in Example 2.6, corresponds to a dilation (shaded) of our Warsaw circle. 
It is easy to see that $\varphi$ is tame on $X$ (with respect to both singular and Čech homology). Moreover, the dimension of the singular persistent homology group $H_{1}^{(u, v)}(X, \varphi)$ is equal to 1 for $v>u>0$ and $v$ sufficiently small, whereas it is equal to 0 when $u=0$, showing that singular persistent homology is not right-continuous in the variable $u$.

Analogously, it is possible to show that simplicial or singular homologies do not ensure the right-continuity in the variable $v$ (see Appendix A).

Let us fix two real numbers $\bar{u}<\bar{v}$ and, for $\bar{u}<u^{\prime} \leq u^{\prime \prime}<\bar{v}$, consider the following commutative diagram

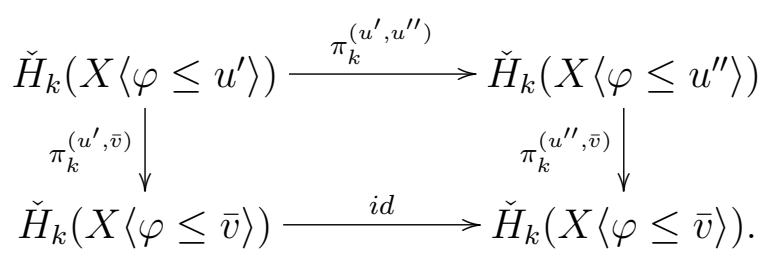

By recalling that $\check{H}_{k}^{(u, v)}(X, \varphi)=\operatorname{im} \pi_{k}^{(u, v)}$, from the above diagram (2.1) it is easy to see that each $\pi_{k}^{\left(u^{\prime}, u^{\prime \prime}\right)}$ induces an injective map $\sigma_{k}^{\left(u^{\prime}, u^{\prime \prime}\right)}: \check{H}_{k}^{\left(u^{\prime}, \bar{v}\right)}(X, \varphi) \rightarrow \check{H}_{k}^{\left(u^{\prime \prime}, \bar{v}\right)}(X, \varphi)$. The following Lemma 2.7 states that, for every $u^{\prime \prime} \geq u^{\prime}>\bar{u}$, with $u^{\prime \prime}$ sufficiently close to $\bar{u}$, the maps $\sigma_{k}^{\left(u^{\prime}, u^{\prime \prime}\right)}$ are all isomorphisms.

Lemma 2.7. Let $(\bar{u}, \bar{v}) \in \Delta^{+}$, and let $\sigma_{k}^{\left(u^{\prime}, u^{\prime \prime}\right)}: \check{H}_{k}^{\left(u^{\prime}, \bar{v}\right)}(X, \varphi) \rightarrow \check{H}_{k}^{\left(u^{\prime \prime}, \bar{v}\right)}(X, \varphi)$ be the injective homomorphism of vector spaces induced by the map $\pi_{k}^{\left(u^{\prime}, u^{\prime \prime}\right)}$. Then there exists $\hat{u}$, with $\bar{u}<\hat{u}<\bar{v}$, such that the maps $\sigma_{k}^{\left(u^{\prime}, u^{\prime \prime}\right)}$ are isomorphisms for every $u^{\prime}, u^{\prime \prime}$ with $\bar{u}<u^{\prime} \leq u^{\prime \prime} \leq \hat{u}$.

Proof. By the Finiteness Theorem 2.3 and the Monotonicity Lemma 2.4, there exists $\hat{u}$, with $\bar{u}<\hat{u}<\bar{v}$, such that $\beta_{\varphi}\left(u^{\prime}, \bar{v}\right)=\operatorname{dim} \check{H}_{k}^{\left(u^{\prime}, \bar{v}\right)}(X, \varphi)$ is finite and equal to $\beta_{\varphi}\left(u^{\prime \prime}, \bar{v}\right)=$ $\operatorname{dim} \check{H}_{k}^{\left(u^{\prime \prime}, \bar{v}\right)}(X, \varphi)$ whenever $\bar{u}<u^{\prime} \leq u^{\prime \prime} \leq \hat{u}$. Since the maps $\sigma_{k}^{\left(u^{\prime}, u^{\prime \prime}\right)}$ are injective (indeed they are inclusions), this implies that they are isomorphisms.

Analogously, by considering the commutative diagram

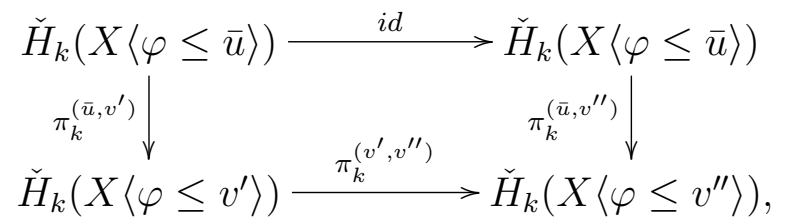

we obtain surjective maps $\tau_{k}^{\left(v^{\prime}, v^{\prime \prime}\right)}: \check{H}_{k}^{\left(\bar{u}, v^{\prime}\right)}(X, \varphi) \rightarrow \check{H}_{k}^{\left(\bar{u}, v^{\prime \prime}\right)}(X, \varphi)$, and prove that they are isomorphisms whenever $v^{\prime}, v^{\prime \prime}$ are sufficiently close to $\bar{v}$, with $\bar{v}<v^{\prime} \leq v^{\prime \prime}$.

Lemma 2.8. Let $(\bar{u}, \bar{v}) \in \Delta^{+}$, and let $\tau_{k}^{\left(v^{\prime}, v^{\prime \prime}\right)}: \check{H}_{k}^{\left(\bar{u}, v^{\prime}\right)}(X, \varphi) \rightarrow \check{H}_{k}^{\left(\bar{u}, v^{\prime \prime}\right)}(X, \varphi)$ be the surjective homomorphism of vector spaces induced by the map $\pi_{k}^{\left(v^{\prime}, v^{\prime \prime}\right)}$. Then there exists 
$\hat{v}>\bar{v}$ such that the homomorphisms $\tau_{k}^{\left(v^{\prime}, v^{\prime \prime}\right)}$ are isomorphisms for every $v^{\prime}, v^{\prime \prime}$ with $\bar{v}<$ $v^{\prime} \leq v^{\prime \prime} \leq \hat{v}$.

Proof. The proof is essentially the same as that of Lemma 2.7, after observing that the maps $\tau_{k}^{\left(v^{\prime}, v^{\prime \prime}\right)}$ are surjections between vector spaces of the same finite dimension.

Proposition 2.9 (Right-Continuity). $\beta_{\varphi}(u, v)$ is right-continuous with respect to both the variables $u$ and $v$.

Proof. In order to prove that $\lim _{u \rightarrow \bar{u}^{+}} \beta_{\varphi}(u, v)=\beta_{\varphi}(\bar{u}, v)$, by the Monotonicity Lemma 2.4 , it will suffice to show that $\check{H}_{k}^{(\bar{u}, \bar{v})}(X, \varphi) \cong \check{H}_{k}^{(\hat{u}, \bar{v})}(X, \varphi)$, where $\hat{u}$ is taken as in Lemma 2.7. To this end, we consider the following sequence of isomorphisms

$$
\begin{aligned}
\check{H}_{k}^{(\bar{u}, \bar{v})}(X, \varphi) & =\operatorname{im} \pi_{k}^{(\bar{u}, \bar{v})} \cong \operatorname{im} \lim _{\leftarrow} \pi_{k}^{\left(u^{\prime}, \bar{v}\right)} \\
& \cong \operatorname{limim}_{\leftarrow} \pi_{k}^{\left(u^{\prime}, \bar{v}\right)}=\lim _{\leftarrow} \check{H}_{k}^{\left(u^{\prime}, \bar{v}\right)}(X, \varphi) \cong \check{H}_{k}^{(\hat{u}, \bar{v})}(X, \varphi) .
\end{aligned}
$$

Let us now show how these equivalences can be obtained.

Let us consider the inverse system of vector spaces $\left(\check{H}_{k}\left(X\left\langle\varphi \leq u^{\prime}\right\rangle\right), \pi_{k}^{\left(u^{\prime}, u^{\prime \prime}\right)}\right)$ over the directed set $\left\{u^{\prime} \in \mathbb{R}: \bar{u}<u^{\prime} \leq \hat{u}\right\}$ decreasingly ordered, and the constant inverse system $\left(\check{H}_{k}(X\langle\varphi \leq \bar{v}\rangle), \pi_{k}^{(\bar{v}, \bar{v})}\right)$, recalling that $\pi_{k}^{(\bar{v}, \bar{v})}$ is the identity.

Clearly the set of homomorphisms $\left\{\pi_{k}^{\left(u^{\prime}, \bar{v}\right)}: \check{H}_{k}\left(X\left\langle\varphi \leq u^{\prime}\right\rangle\right) \rightarrow \check{H}_{k}(X\langle\varphi \leq \bar{v}\rangle)\right\}$ is a homomorphism of inverse systems because the diagram

is commutative.

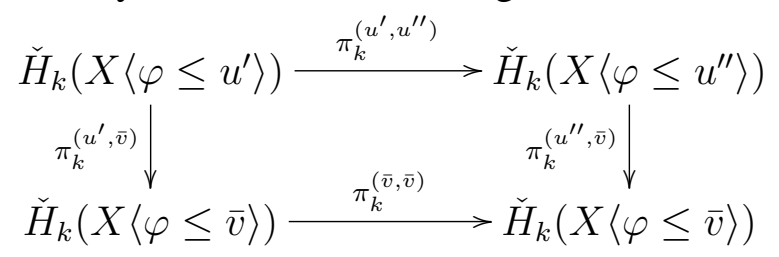

By the continuity of Čech Theory (cf. [31, Thm. X, 3.1]), $\lim _{\leftarrow} \check{H}_{k}\left(X\left\langle\varphi \leq u^{\prime}\right\rangle\right)=$ $\check{H}_{k}(X\langle\varphi \leq \bar{u}\rangle)$. Obviously, $\lim _{\leftarrow} \check{H}_{k}(X\langle\varphi \leq \bar{v}\rangle)=\check{H}_{k}(X\langle\varphi \leq \bar{v}\rangle)$. Hence, $\pi_{k}^{(\bar{u}, \bar{v})}$ is the natural homomorphism $\lim _{\leftarrow} \pi_{k}^{\left(\overleftarrow{u^{\prime}}, \bar{v}\right)}$ between the inverse limits. So, im $\pi_{k}^{(\bar{u}, \bar{v})} \cong \operatorname{im} \lim _{\leftarrow} \pi_{k}^{\left(u^{\prime}, \bar{v}\right)}$.

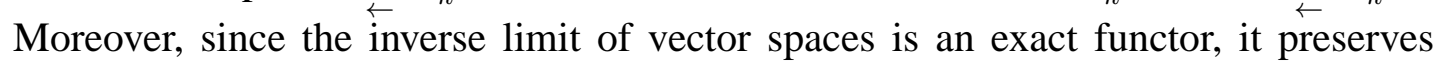
epimorphisms and hence images. Therefore, it holds that $\operatorname{im}_{\leftarrow} \pi_{k}^{\left(u^{\prime}, \bar{v}\right)} \cong \lim _{\leftarrow} \operatorname{im} \pi_{k}^{\left(u^{\prime}, \bar{v}\right)}=$ $\lim \check{H}_{k}^{\left(u^{\prime}, \bar{v}\right)}(X, \varphi)$, where the last inverse limit is taken with respect to the inverse system $\left.\overleftarrow{(}_{k}^{\left(u^{\prime}, \bar{v}\right)}(X, \varphi), \sigma_{k}^{\left(u^{\prime}, u^{\prime \prime}\right)}\right)$ over the directed set $\left\{u^{\prime} \in \mathbb{R}: \bar{u}<u^{\prime} \leq \hat{u}\right\}$ decreasingly ordered, and $\sigma_{k}^{\left(u^{\prime}, u^{\prime \prime}\right)}$ are the maps introduced in Lemma 2.7.

Finally, $\lim _{\leftarrow} \check{H}_{k}^{\left(u^{\prime}, \bar{v}\right)}(X, \varphi) \cong \check{H}_{k}^{(\hat{u}, \bar{v})}(X, \varphi)$. Indeed, $\lim _{\leftarrow} \check{H}_{k}^{\left(u^{\prime}, \bar{v}\right)}(X, \varphi)$ is the inverse limit of a system of isomorphic vector spaces by Lemma $\overleftarrow{2.7}$

Analogously for the variable $v$, applying Lemma 2.8 . 


\section{Stability of one-dimensional PBNs}

In this section we prove the stability of PBNs for continuous scalar-valued filtering functions (Theorem 3.13). This result generalizes the main theorem in [20], which requires tame functions on triangulable spaces. Our proof relies on a number of basic simple properties of PBNs that are completely analogous to those proved in $[23,33]$ and used to show the PBNs stability in the case of the 0th homology. For this reason we shall omit the proofs of our statements when they are quite analogous to already published ones. Some of these properties, such as those needed to introduce persistence diagrams, require the finiteness of PBNs proved in the previous section. Along the way we shall also prove the Representation Theorem 3.11. It guarantees that one-dimensional PBNs are completely determined by persistence diagrams, even in the case of just continuous functions.

The following Lemmas 3.1 and 3.2 can be proved in the same way as the analogous results holding when the homology degree $k$ is equal to 0 (see [23]).

Lemma 3.1. The following statements hold:

(i) For every $u<\min \varphi, \beta_{\varphi}(u, v)=0$.

(ii) For every $v \geq \max \varphi, \beta_{\varphi}(u, v)$ is equal to the maximum number of linearly independent classes in $\check{H}_{k}(X)$ having at least one representative in $X\langle\varphi \leq u\rangle$.

We observe that Lemma 3.1 (ii) implies that, for every $v \geq \max \varphi, \beta_{\varphi}(u, v)$ is independent of $v$.

Since, for $u_{1} \leq u_{2}<v_{1} \leq v_{2}$, the maximum number of linearly independent homology classes born between $u_{1}$ and $u_{2}$ and still linearly independent at $v_{1}$ is certainly not smaller than the maximum number of those still linearly independent at $v_{2}$, we have the next result.

Lemma 3.2 (Jump Monotonicity). Let $u_{1}, u_{2}, v_{1}, v_{2}$ be real numbers such that $u_{1} \leq u_{2}<$ $v_{1} \leq v_{2}$. It holds that

$$
\beta_{\varphi}\left(u_{2}, v_{1}\right)-\beta_{\varphi}\left(u_{1}, v_{1}\right) \geq \beta_{\varphi}\left(u_{2}, v_{2}\right)-\beta_{\varphi}\left(u_{1}, v_{2}\right)
$$

Lemma 3.2 justifies the following definitions of multiplicity. Since we are working with continuous instead of tame functions, we adopt the definitions introduced in [33] rather than those of [20]. Although based on the same idea, the difference relies on the computation of multiplicity on a varying grid, instead of a fixed one. So we can work with an infinite number of (possibly accumulating) points with a positive multiplicity. Due to the lack of a well-established terminology for points with a positive multiplicity, we call them cornerpoints, as in previous papers. 
Definition 3.3 (Proper cornerpoint). For every point $p=(u, v) \in \Delta^{+}$, we define the number $\mu(p)$ as the minimum over all the positive real numbers $\varepsilon$, with $u+\varepsilon<v-\varepsilon$, of

$$
\beta_{\varphi}(u+\varepsilon, v-\varepsilon)-\beta_{\varphi}(u-\varepsilon, v-\varepsilon)-\beta_{\varphi}(u+\varepsilon, v+\varepsilon)+\beta_{\varphi}(u-\varepsilon, v+\varepsilon) .
$$

The number $\mu(p)$ will be called the multiplicity of $p$ for $\beta_{\varphi}$. Moreover, we shall call a proper cornerpoint for $\beta_{\varphi}$ any point $p \in \Delta^{+}$such that the number $\mu(p)$ is strictly positive.

Definition 3.4 (Cornerpoint at infinity). For every vertical line $r$, with equation $u=\bar{u}$, $\bar{u} \in \mathbb{R}$, let us identify $r$ with $(\bar{u}, \infty) \in \Delta^{*}$, and define the number $\mu(r)$ as the minimum over all the positive real numbers $\varepsilon$, with $\bar{u}+\varepsilon<1 / \varepsilon$, of

$$
\beta_{\varphi}\left(\bar{u}+\varepsilon, \frac{1}{\varepsilon}\right)-\beta_{\varphi}\left(\bar{u}-\varepsilon, \frac{1}{\varepsilon}\right) .
$$

The number $\mu(r)$ will be called the multiplicity of $r$ for $\beta_{\varphi}$. When this finite number is strictly positive, we call $r$ a cornerpoint at infinity for $\beta_{\varphi}$.

The concept of cornerpoint allows us to introduce a representation of the PBNs, based on the following definition [20].

Definition 3.5 (Persistence diagram). The persistence diagram $D_{\varphi}$ is the multiset of all cornerpoints (both proper and at infinity) for $\beta_{\varphi}$, counted with their multiplicity, union the points of $\Delta$, counted with infinite multiplicity.

In order to show that persistence diagrams completely describe PBNs, we give some technical results concerning cornerpoints.

The Monotonicity Lemma 2.4, the Right-Continuity Proposition 2.9 and the Jump Monotonicity Lemma 3.2 imply the following result, by the same arguments as in [33].

Proposition 3.6 (Propagation of Discontinuities). If $\bar{p}=(\bar{u}, \bar{v})$ is a proper cornerpoint for $\beta_{\varphi}$, then the following statements hold:

(i) If $\bar{u} \leq u<\bar{v}$, then $\bar{v}$ is a discontinuity point for $\beta_{\varphi}(u, \cdot)$;

(ii) If $\bar{u}<v<\bar{v}$, then $\bar{u}$ is a discontinuity point for $\beta_{\varphi}(\cdot, v)$.

If $\bar{r}=(\bar{u}, \infty)$ is a cornerpoint at infinity for $\beta_{\varphi}$, then it holds that

(iii) If $\bar{u}<v$, then $\bar{u}$ is a discontinuity point for $\beta_{\varphi}(\cdot, v)$.

We observe that any open arcwise connected neighborhood in $\Delta^{+}$of a discontinuity point for $\beta_{\varphi}$ contains at least one discontinuity point in the variable $u$ or $v$. Moreover, as a consequence of the Jump Monotonicity Lemma 3.2, discontinuity points in the variable $u$ propagate downwards, while discontinuity points in the variable $v$ propagate rightwards. So, by applying the Finiteness Theorem 2.3, we obtain the proposition below (cf. [33, Prop. 6]). 
Proposition 3.7. For every point $\bar{p}=(\bar{u}, \bar{v}) \in \Delta^{+}$, a real number $\varepsilon>0$ exists such that the open set

$$
W_{\varepsilon}(\bar{p})=\left\{(u, v) \in \mathbb{R}^{2}:|u-\bar{u}|<\varepsilon,|v-\bar{v}|<\varepsilon, u \neq \bar{u}, v \neq \bar{v}\right\}
$$

is contained in $\Delta^{+}$, and does not contain any discontinuity point for $\beta_{\varphi}$.

As a simple consequence of Lemma 3.1 and Definition 3.3, we have the following proposition.

Proposition 3.8 (Localization of Cornerpoints). If $\bar{p}=(\bar{u}, \bar{v})$ is a proper cornerpoint for $\beta_{\varphi}$, then $\bar{p} \in\left\{(u, v) \in \Delta^{+}: \min \varphi \leq u<v \leq \max \varphi\right\}$.

By applying Propositions 3.6, 3.7, and 3.8 it is easy to prove the following result.

Proposition 3.9 (Local Finiteness of Cornerpoints). For each strictly positive real number $\varepsilon, \beta_{\varphi}$ has, at most, a finite number of cornerpoints in $\left\{(u, v) \in \mathbb{R}^{2}: u+\varepsilon<v\right\}$.

We observe that it is easy to provide examples of persistence diagrams containing an infinite number of proper cornerpoints, accumulating onto the diagonal $\Delta$.

Remark 3.10. The number of cornerpoints at infinity for $\beta_{\varphi}$ counted with their multiplicities is equal to $\operatorname{dim} \check{H}_{k}(X)$, and hence it is finite and independent of $\varphi$.

The following Theorem 3.11 shows that persistence diagrams uniquely determine onedimensional PBNs (the inverse also holds by definition of persistence diagram). We remark that a similar result was given in [20], under the name of k-triangle Lemma. Our Representation Theorem differs from the $k$-triangle Lemma in two respects. Firstly, our assumptions on the function $\varphi$ are weaker. Secondly, the $k$-triangle Lemma focuses not on all the set $\Delta^{+}$, but only on the points with coordinates that are not homological critical values.

Theorem 3.11 (Representation Theorem). For every $(\bar{u}, \bar{v}) \in \Delta^{+}$, we have

$$
\beta_{\varphi}(\bar{u}, \bar{v})=\sum_{\substack{(u, v) \in \Delta^{*} \\ u \leq \bar{u}, v>\bar{v}}} \mu((u, v)) .
$$

Proof. The claim is a consequence of the definitions of multiplicity (Definitions 3.3 and 3.4), together with the previous results about cornerpoints, and the Right-Continuity Proposition 2.9, in the same way as done in [33].

As a consequence of the Representation Theorem 3.11, any distance between persistence diagrams induces a distance between one-dimensional PBNs. This justifies the following definition [23]. 
Definition 3.12 (Matching distance). Let $X, Y$ be triangulable spaces endowed with continuous functions $\varphi: X \rightarrow \mathbb{R}, \psi: Y \rightarrow \mathbb{R}$. The (extended) matching distance $d_{\text {match }}$ between $\beta_{\varphi}$ and $\beta_{\psi}$ is defined by

$$
d_{\text {match }}\left(\beta_{\varphi}, \beta_{\psi}\right)=\inf _{\gamma} \sup _{p \in D_{\varphi}}\|p-\gamma(p)\|_{\widetilde{\infty}}
$$

where $\gamma$ ranges over all multi-bijections between $D_{\varphi}$ and $D_{\psi}$, and, for every $p=(u, v), q=$ $\left(u^{\prime}, v^{\prime}\right)$ in $\bar{\Delta}^{*}$,

$$
\|p-q\|_{\widetilde{\infty}}=\min \left\{\max \left\{\left|u-u^{\prime}\right|,\left|v-v^{\prime}\right|\right\}, \max \left\{\frac{v-u}{2}, \frac{v^{\prime}-u^{\prime}}{2}\right\}\right\},
$$

with the convention about points at infinity that $\infty-y=y-\infty=\infty$ when $y \neq \infty$, $\infty-\infty=0, \frac{\infty}{2}=\infty,|\infty|=\infty, \min \{c, \infty\}=c$ and $\max \{c, \infty\}=\infty$.

In plain words, $\|\cdot\|_{\widetilde{\infty}}$ measures the pseudo-distance between two points $p$ and $q$ as the minimum between the cost of moving one point onto the other and the cost of moving both points onto the diagonal, with respect to the max-norm and under the assumption that any two points of the diagonal have vanishing pseudo-distance.

The term extended means that $d_{\text {match }}$ can take the value $+\infty$. It will follow from our One-Dimensional Stability Theorem 3.13 that $d_{\text {match }}$ is finite when $X=Y$.

When the number of cornerpoints is finite, the matching of persistence diagrams is related to the bottleneck transportation problem, and the matching distance reduces to the bottleneck distance [20]. In our case, however, the number of cornerpoints may be countably infinite, because of our loose assumption on the filtering function, that is only required to be continuous.

We observe that, although the number of cornerpoints may be countably infinite, in (3.1) we can write max instead of sup and min instead of inf, as can be formally proven using the same arguments as in [23, Thm. 28]. In other words, a multi-bijection $\bar{\gamma}$ exists for which $d_{\text {match }}\left(\beta_{\varphi}, \beta_{\psi}\right)=\max _{p \in D_{\varphi}}\|p-\bar{\gamma}(p)\|_{\widetilde{\infty}}$. Every such matching will henceforth be called optimal.

We are now ready to give the one-dimensional stability theorem for PBNs with continuous filtering functions. The proof relies on a cone construction. The rationale behind this construction is to directly apply the arguments used in [23], eliminating cornerpoints at infinity, whose presence would require us to modify all the proofs.

This stability theorem is a different result from the one given in [20], weakening the tameness requirement to continuity, and actually solving one of the open problems posed in that work by the authors.

Theorem 3.13 (One-Dimensional Stability Theorem). Let $X$ be a triangulable space, and $\varphi, \psi: X \rightarrow \mathbb{R}$ two continuous functions. Then $d_{\text {match }}\left(\beta_{\varphi}, \beta_{\psi}\right) \leq \max _{x \in X}|\varphi(x)-\psi(x)|$. 
Proof. In what follows we can assume that $X$ is connected. Indeed, if $X$ has $r$ connected components $C_{1}, \ldots, C_{r}$, then the claim can be proved by induction after observing that $D_{\varphi}=\bigcup_{i=1}^{r} D_{\varphi_{\mid C_{i}}}$.

For the 0th homology, the claim has been proved in [23, Thm. 25].

Let us now consider the $k$ th homology with $k>0$. We build the cone on $X, \tilde{X}=$ $(X \times I) /(X \times\{1\})$ (see Figure 2).

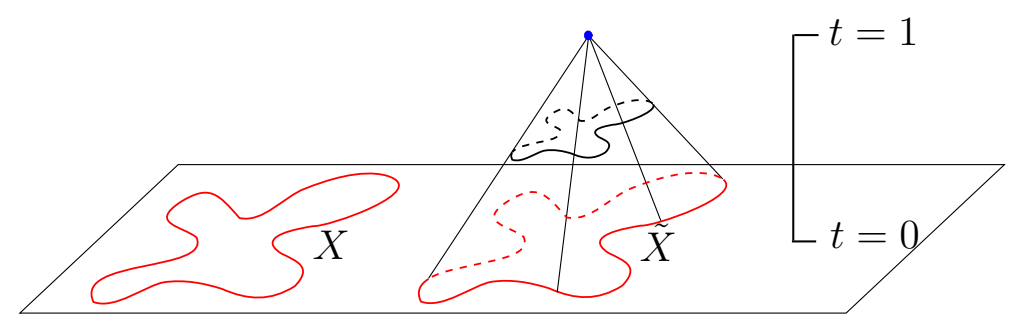

Figure 2: The cone construction used in the proof of Theorem 3.13. The cycles in the cone are null-homologous.

Since $X$ is triangulable, so is $\tilde{X}$. We also consider the continuous function $\tilde{\varphi}: \tilde{X} \rightarrow \mathbb{R}$ taking the class of $(x, t)$ in $\tilde{X}$ to the value $\varphi(x) \cdot(1-t)+M \cdot t$, where $M=3$. $(\max |\varphi|+\max |\psi|)+1$. This choice of $M$, besides guaranteeing that $(u, M) \in \Delta^{+}$ when $u \leq \max |\varphi|, \max |\psi|$, will be useful later.

By construction, it holds that

$$
\beta_{\tilde{\varphi}}(u, v)= \begin{cases}\beta_{\varphi}(u, v), & \text { if } v<M \\ 0, & \text { if } v \geq M\end{cases}
$$

Indeed, it is well known that $\tilde{X}$ is contractible (see [34, Lemma 21.13]), explaining why $\beta_{\tilde{\varphi}}(u, v)=0$ when $v \geq M$. The other case $v<M$ follows from the observation that, for every $v<M$, identifying $X\langle\varphi \leq v\rangle \times\{0\}$ with $X\langle\varphi \leq v\rangle$, the lower level set $X\langle\varphi \leq v\rangle$ is a strong deformation retract of $\tilde{X}\langle\tilde{\varphi} \leq v\rangle$. To see this, it is sufficient to consider the obvious retraction $r:(x, t) \mapsto x$ and the deformation retraction $H: \tilde{X}\langle\tilde{\varphi} \leq v\rangle \times I \rightarrow$ $\tilde{X}\langle\tilde{\varphi} \leq v\rangle, H((x, t), s)=(x, t \cdot(1-s))$. This yields the following commutative diagram

$$
\begin{gathered}
\check{H}_{k}(\tilde{X}\langle\tilde{\varphi} \leq u\rangle) \stackrel{r_{k}^{\prime}}{\longrightarrow} \check{H}_{k}(X\langle\varphi \leq u\rangle) \\
\tilde{\pi}_{k}^{(u, v)} \downarrow \\
\check{H}_{k}(\tilde{X}\langle\tilde{\varphi} \leq v\rangle) \stackrel{{ }_{k}^{\prime \prime}}{\longrightarrow} \check{H}_{k}(X\langle\varphi \leq v\rangle),
\end{gathered}
$$

where the horizontal maps are isomorphisms induced by $r$, and the vertical ones are the homomorphisms introduced in Definition 2.1 for the respective spaces. So, $\check{H}_{k}^{(u, v)}(\tilde{X}, \tilde{\varphi}) \cong$ $\check{H}_{k}^{(u, v)}(X, \varphi)$ when $v<M$.

Clearly, a point of $\Delta^{+}$is a proper cornerpoint for $\beta_{\varphi}$ if and only if it is a proper cornerpoint for $\beta_{\tilde{\varphi}}$, with the ordinate strictly less than $M$. Moreover, a point $(u, \infty)$ of 
$\Delta^{*}$ is a cornerpoint at infinity for $\beta_{\varphi}$ if and only if the point $(u, M) \in \Delta^{+}$is a proper cornerpoint for $\beta_{\tilde{\varphi}}$. We remark that there are no cornerpoints $(u, v)$ for $\beta_{\tilde{\varphi}}$ when $\max |\varphi|<$ $v<M$.

Analogously, we can construct $\tilde{\psi}: \tilde{X} \rightarrow \mathbb{R}$ out of $\psi$ with the same properties.

It is possible to prove the inequality $d_{\text {match }}\left(\beta_{\tilde{\varphi}}, \beta_{\tilde{\psi}}\right) \leq \max _{\tilde{x} \in \tilde{X}}|\tilde{\varphi}(\tilde{x})-\tilde{\psi}(\tilde{x})|$. Since the proof of this claim is completely analogous to that of [23, Thm. 25], we omit the technical details remarking that it is precisely here that Lemma 2.5, and Propositions 3.6, 3.7, 3.8 and 3.9 are needed. Hence, since $\max _{\tilde{x} \in \tilde{X}}|\tilde{\varphi}(\tilde{x})-\tilde{\psi}(\tilde{x})|=\max _{x \in X}|\varphi(x)-\psi(x)|$, it is sufficient to show that $d_{\text {match }}\left(\beta_{\varphi}, \beta_{\psi}\right) \leq d_{\text {match }}\left(\beta_{\tilde{\varphi}}, \beta_{\tilde{\psi}}\right)$.

To this end, we can consider an optimal matching $\tilde{\gamma}$ between $D_{\tilde{\varphi}}$ and $D_{\tilde{\psi}}$, for which $d_{\text {match }}\left(\beta_{\tilde{\varphi}}, \beta_{\tilde{\psi}}\right)=\max _{\tilde{p} \in D_{\tilde{\varphi}}}\|\tilde{p}-\tilde{\gamma}(\tilde{p})\|_{\tilde{\infty}}$. Since $\tilde{\gamma}$ is optimal, $\tilde{\gamma}$ takes each point $(u, v) \in$ $D_{\tilde{\varphi}}$, with $v=M$, to a point $\left(u^{\prime}, v^{\prime}\right) \in D_{\tilde{\psi}}$, with $v^{\prime}=M$. Indeed, if it were not true, i.e. $\tilde{\gamma}((u, M))=\left(u^{\prime}, v^{\prime}\right)$ with $v^{\prime}<M$, then $v^{\prime} \leq \max |\psi|$, since there are no cornerpoints $\left(u^{\prime}, v^{\prime}\right)$ for $\beta_{\tilde{\psi}}$ with $\max |\psi|<v^{\prime}<M$. By the choice of $M$, we would have $\|(u, M)-\tilde{\gamma}((u, M))\|_{\widetilde{\infty}}>\max _{x \in X}|\varphi(x)-\psi(x)|$. This contradicts $d_{\text {match }}\left(\beta_{\tilde{\varphi}}, \beta_{\tilde{\psi}}\right) \leq$ $\max _{x \in X}|\varphi(x)-\psi(x)|$. The same argument holds for $\tilde{\gamma}^{-1}$, and this proves that $\tilde{\gamma}$ maps cornerpoints whose ordinate is smaller than $M$ into cornerpoints whose ordinate is still below $M$.

We now show that there exists a multi-bijection $\gamma$ between $D_{\varphi}$ and $D_{\psi}$, such that $\max _{p \in D_{\varphi}}\|p-\gamma(p)\|_{\widetilde{\infty}}=\max _{\tilde{p} \in D_{\tilde{\varphi}}}\|\tilde{p}-\tilde{\gamma}(\tilde{p})\|_{\widetilde{\infty}}$, thus proving that $d_{\text {match }}\left(\beta_{\varphi}, \beta_{\psi}\right) \leq$ $d_{\text {match }}\left(\beta_{\tilde{\varphi}}, \beta_{\tilde{\psi}}\right)$. Indeed, we can define $\gamma: D_{\varphi} \rightarrow D_{\psi}$ by setting $\gamma((u, v))=\tilde{\gamma}((u, v))$ if $v<\infty$, and $\gamma((u, v))=\left(u^{\prime}, v\right)$, where $u^{\prime}$ is the abscissa of the point $\tilde{\gamma}((u, M))$, if $v=\infty$. This concludes the proof.

Let us conclude this section by showing the following property of $d_{\text {match }}$, that will be useful later.

Proposition 3.14. Let $\lambda \in \mathbb{R}$, with $\lambda>0$. Let also $\varphi: X \rightarrow \mathbb{R}, \psi: Y \rightarrow \mathbb{R}$ be two continuous filtering functions for the triangulable spaces $X$ and $Y$, respectively. Then, it holds that

$$
d_{\text {match }}\left(\beta_{\lambda \cdot \varphi}, \beta_{\lambda \cdot \psi}\right)=\lambda \cdot d_{\text {match }}\left(\beta_{\varphi}, \beta_{\psi}\right)
$$

Proof. First of all, let us observe that, if $(u, v) \in \Delta^{+}$, then $(\lambda u, \lambda v) \in \Delta^{+}$for $\lambda>0$. Moreover, for $\lambda>0$, it holds that $\beta_{\varphi}(u, v)=\beta_{\lambda \cdot \varphi}(\lambda u, \lambda v)$, since $X\langle\varphi \leq u\rangle=X\langle\lambda$. $\varphi \leq \lambda u\rangle$ for every $u \in \mathbb{R}$. Then, by Definitions 3.3 and 3.4, for every $(u, v) \in \Delta^{*}$, the multiplicity of $(u, v)$ for $\beta_{\varphi}$ is equal to the multiplicity of $(\lambda u, \lambda v)$ for $\beta_{\lambda \cdot \varphi}$.

Following the definition of the operator $\|\cdot\|_{\widetilde{\infty}}$ in Definition 3.12, for every $(u, v),\left(u^{\prime}, v^{\prime}\right)$ 
in $\Delta^{*}$, we have

$$
\begin{aligned}
& \left\|(\lambda u, \lambda v)-\left(\lambda u^{\prime}, \lambda v^{\prime}\right)\right\|_{\widetilde{\infty}}= \\
& =\min \left\{\max \left\{\lambda\left|u-u^{\prime}\right|, \lambda\left|v-v^{\prime}\right|\right\}, \max \left\{\lambda \frac{v-u}{2}, \lambda \frac{v^{\prime}-u^{\prime}}{2}\right\}\right\} \\
& =\lambda\left\|(u, v)-\left(u^{\prime}, v^{\prime}\right)\right\|_{\widetilde{\infty}} .
\end{aligned}
$$

Thus the claim follows from the definition of $d_{\text {match }}$ (Definition 3.12), and by observing that the correspondence taking each pair $(u, v) \in \Delta^{*}$ to the pair $(\lambda u, \lambda v) \in \Delta^{*}$ is actually a bijection.

\section{Stability of multidimensional PBNs}

We now provide the proof of the stability of multidimensional PBNs. It will be deduced following the same arguments given in [16] to prove the stability of multidimensional PBNs for the case of the 0th homology.

The key idea is that a foliation in half-planes of $\Delta^{+}$can be given, such that the restriction of the multidimensional PBNs function to these half-planes turns out to be a one-dimensional PBNs function in two scalar variables. This approach implies that the comparison of two multidimensional PBNs functions can be performed leaf by leaf by measuring the distance of appropriate one-dimensional PBNs functions. Therefore, the stability of multidimensional persistence is a consequence of the one-dimensional persistence stability.

We start by recalling that the following parameterized family of half-planes in $\mathbb{R}^{n} \times \mathbb{R}^{n}$ is a foliation of $\Delta^{+}$(cf. [16, Prop. 1]).

Definition 4.1 (Admissible pairs). For every vector $\vec{l}=\left(l_{1}, \ldots, l_{n}\right)$ of $\mathbb{R}^{n}$ such that $l_{i}>0$ for $i=1, \ldots, n$, and $\sum_{i=1}^{n} l_{i}^{2}=1$, and for every vector $\vec{b}=\left(b_{1}, \ldots, b_{n}\right)$ of $\mathbb{R}^{n}$ such that $\sum_{i=1}^{n} b_{i}=0$, we shall say that the pair $(\vec{l}, \vec{b})$ is admissible. We shall denote the set of all admissible pairs in $\mathbb{R}^{n} \times \mathbb{R}^{n}$ by $A d m_{n}$. Given an admissible pair $(\vec{l}, \vec{b})$, we define the half-plane $\pi_{(\vec{l}, \vec{b})}$ of $\mathbb{R}^{n} \times \mathbb{R}^{n}$ by the following parametric equations:

$$
\left\{\begin{array}{l}
\vec{u}=s \vec{l}+\vec{b} \\
\vec{v}=t \vec{l}+\vec{b}
\end{array}\right.
$$

for $s, t \in \mathbb{R}$, with $s<t$.

Since these half-planes $\pi_{(\vec{l}, \vec{b})}$ constitute a foliation of $\Delta^{+}$, for each $(\vec{u}, \vec{v}) \in \Delta^{+}$there exists one and only one $(\vec{l}, \vec{b}) \in A d m_{n}$ such that $(\vec{u}, \vec{v}) \in \pi_{(\vec{l}, \vec{b})}$. Observe that $\vec{l}$ and $\vec{b}$ only depend on $(\vec{u}, \vec{v})$. 
A first property of this foliation is that the restriction of $\beta_{\vec{\varphi}}$ to each leaf can be seen as a particular one-dimensional PBNs function. Intuitively, on each half plane $\pi_{(\vec{l}, \vec{b})}$ one can find the PBNs corresponding to the filtration of $X$ obtained by sweeping the line through $\vec{u}$ and $\vec{v}$ parameterized by $\gamma_{(\vec{l}, \vec{b})}: \mathbb{R} \rightarrow \mathbb{R}^{n}$, with $\gamma_{(\vec{l}, \vec{b})}(\tau)=\tau \vec{l}+\vec{b}$.

A second property is that this filtration corresponds to the one given by the lower level sets of a certain scalar-valued continuous function. Both these properties are stated in the next theorem, analogous to [17, Thm. 2], and are intuitively shown in Figure 3.

Theorem 4.2 (Reduction Theorem). For every $(\vec{u}, \vec{v}) \in \Delta^{+}$, let $(\vec{l}, \vec{b})$ be the only admissible pair such that $(\vec{u}, \vec{v})=(s \vec{l}+\vec{b}, t \vec{l}+\vec{b}) \in \pi_{(\vec{l}, \vec{b})}$. Let moreover $\varphi_{(\vec{u}, \vec{v})}: X \rightarrow \mathbb{R}$ be the continuous filtering function defined by setting

$$
\varphi_{(\vec{u}, \vec{v})}(x)=\min _{i} l_{i} \cdot \max _{i} \frac{\varphi_{i}(x)-b_{i}}{l_{i}} .
$$

Then $X\langle\vec{\varphi} \preceq \vec{u}\rangle=X\left\langle\left(\min _{i} l_{i}\right)^{-1} \varphi_{(\vec{u}, \vec{v})} \leq s\right\rangle$. Therefore

$$
\beta_{\vec{\varphi}}(\vec{u}, \vec{v})=\beta_{\left(\min _{i} l_{i}\right)^{-1} \varphi_{(\vec{u}, \vec{v})}}(s, t) .
$$

Proof. For every $\vec{u}=\left(u_{1}, \ldots, u_{n}\right) \in \mathbb{R}^{n}$, with $u_{i}=s l_{i}+b_{i}, i=1, \ldots, n, s \in \mathbb{R}$, the following equalities hold:

$$
\begin{aligned}
X\langle\vec{\varphi} \preceq \vec{u}\rangle & =\left\{x \in X: \varphi_{i}(x) \leq u_{i}, i=1, \ldots, n\right\} \\
& =\left\{x \in X: \max _{i} \frac{\varphi_{i}(x)-b_{i}}{l_{i}} \leq s\right\}=\left\{x \in X:\left(\min _{i} l_{i}\right)^{-1} \varphi_{(\vec{u}, \vec{v})} \leq s\right\} \\
& =X\left\langle\left(\min _{i} l_{i}\right)^{-1} \varphi_{(\vec{u}, \vec{v})} \leq s\right\rangle .
\end{aligned}
$$

The last claim follows from the definition of PBNs.

Definition 4.1 and Theorem 4.2 might appear unnecessarily cumbersome. A naive idea for proving stability via the one-dimensional reduction might be to directly apply the onedimensional theory to the line through $\vec{u}$ and $\vec{v}$. This does not work without introducing the functions $\varphi_{(\vec{u}, \vec{v})}$ and without a one-dimensional stability result for continuous filtering functions such as our Theorem 3.13. Indeed, an analogous result for tame functions would not be applicable in our case since, as remarked in [17], the set of tame functions is not closed under the maximum operator.

Finally, the most important property of our foliation is that it allows us to obtain an analogue of the distance $d_{\text {match }}$ for the multidimensional case, denoted by $D_{\text {match }}$, having a particularly simple form, yet yielding the desired stability result.

Our definition of $D_{\text {match }}$ is the natural one in order to compare multidimensional PBNs. Indeed, it boils down to matching cornerpoints of $\vec{\varphi}$ that arise from the onedimensional filtration obtained sweeping the line through $\vec{u}$ and $\vec{v}$ with those of $\vec{\psi}$ along 

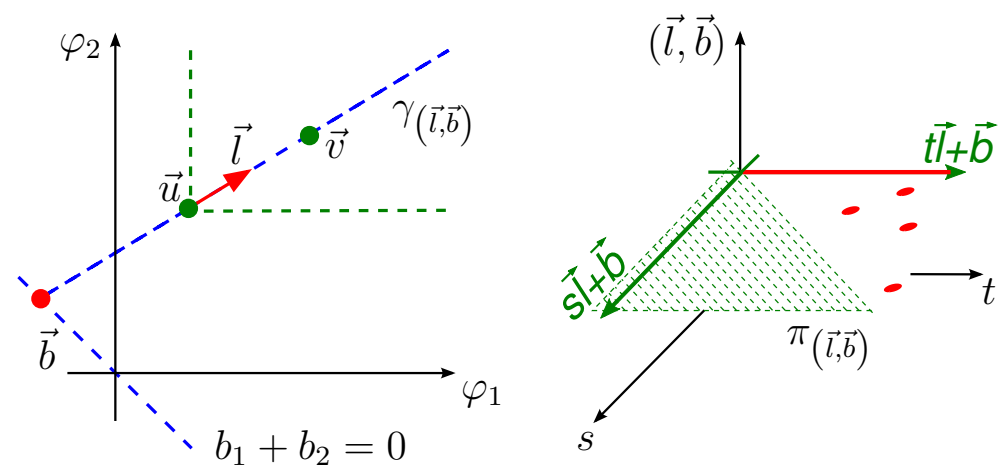

Figure 3: One-dimensional reduction of two-dimensional PBNs. Left: a one-dimensional filtration is constructed sweeping the line through $\vec{u}$ and $\vec{v}$. A unit vector $\vec{l}$ and a point $\vec{b}$ are used to parameterize this line as $\gamma_{(\vec{l}, \vec{b})}(\tau)=\tau \vec{l}+\vec{b}$. Right: the persistence diagram of this filtration can be found on the leaf $\pi_{(\vec{l}, \vec{b})}$ of the foliation.

the same line. In our treatment this is accomplished using a particular parameterization of this line. However this parameterization is not mandatory: Other parameterizations of the same line would yield the same distance, as it has been proved in [35]. In other words, $D_{\text {match }}$ is in some way intrinsically defined.

$D_{\text {match }}$ was introduced in [17] (see also [16]), although in the narrower setting of max-tame filtering functions, and can be rewritten as follows.

Definition 4.3 (Multidimensional matching distance). Let $X, Y$ be triangulable spaces endowed with continuous functions $\vec{\varphi}: X \rightarrow \mathbb{R}^{n}, \vec{\psi}: Y \rightarrow \mathbb{R}^{n}$. The (extended) multidimensional matching distance $D_{\text {match }}$ between $\beta_{\vec{\varphi}}$ and $\beta_{\vec{\psi}}$ is defined as

$$
D_{\text {match }}\left(\beta_{\vec{\varphi},}, \beta_{\vec{\psi}}\right)=\sup _{(\vec{u}, \vec{v}) \in \Delta^{+}} d_{\text {match }}\left(\beta_{\left.\varphi_{(\vec{u}, \vec{v}}\right)}, \beta_{\psi_{(\vec{u}, \vec{v})}}\right) .
$$

The following theorem shows not only that $D_{\text {match }}$ is a distance when $X=Y$, but, more importantly, the stability of multidimensional PBNs with respect to this distance.

Theorem 4.4 (Multidimensional Stability Theorem). If $X$ is a triangulable space, then $D_{\text {match }}$ is a distance on the set $\left\{\beta_{\vec{\varphi}} \mid \vec{\varphi}: X \rightarrow \mathbb{R}^{n}\right.$ continuous $\}$. Moreover,

$$
D_{\text {match }}\left(\beta_{\vec{\varphi}}, \beta_{\vec{\psi}}\right) \leq \max _{x \in X}\|\vec{\varphi}(x)-\vec{\psi}(x)\|_{\infty} .
$$

Proof. Let us begin by observing that

$$
\begin{aligned}
d_{\text {match }}\left(\beta_{\left.\varphi_{(\vec{u}, \vec{v}}\right)}, \beta_{\psi_{(\vec{u}, \vec{v})}}\right) & \leq \max _{x \in X}\left|\varphi_{(\vec{u}, \vec{v})}(x)-\psi_{(\vec{u}, \vec{v})}(x)\right| \\
& \leq \min _{i} l_{i} \cdot \max _{x \in X}\left|\max _{i} \frac{\varphi_{i}(x)-b_{i}}{l_{i}}-\max _{i} \frac{\psi_{i}(x)-b_{i}}{l_{i}}\right| \\
& \leq \min _{i} l_{i} \cdot \frac{\max _{x \in X}|| \vec{\varphi}(x)-\left.\vec{\psi}(x)\right|_{\infty}}{\min _{i} l_{i}}
\end{aligned}
$$


where the first inequality follows applying the One-Dimensional Stability Theorem 3.13, since $\varphi_{(\vec{u}, \vec{v})}, \psi_{(\vec{u}, \vec{v})}$ are scalar-valued continuous filtering functions, while the second inequality descends from the definition of $\varphi_{(\vec{u}, \vec{v})}$ and $\psi_{(\vec{u}, \vec{v})}$ (cf. Theorem 4.2). Therefore, $D_{\text {match }}\left(\beta_{\vec{\varphi}}, \beta_{\vec{\psi}}\right)$ is bounded by $\max _{x \in X}\|\vec{\varphi}(x)-\vec{\psi}(x)\|_{\infty}$.

Let us now prove that $D_{\text {match }}$ is a distance on $\left\{\beta_{\vec{\varphi}} \mid \vec{\varphi}: X \rightarrow \mathbb{R}^{n}\right.$ continuous $\}$. Since $D_{\text {match }}$ is bounded, it takes values in the set of non-negative reals. Moreover, as a consequence of the Reduction Theorem 4.2, the identity $\beta_{\vec{\varphi}} \equiv \beta_{\vec{\psi}}$ holds if and only if $\beta_{\left(\min _{i} l_{i}\right)^{-1} \varphi_{(\vec{u}, \vec{v})}} \equiv \beta_{\left(\min _{i} l_{i}\right)^{-1} \psi_{(\vec{u}, \vec{v})}}$ for every $(\vec{u}, \vec{v}) \in \Delta^{+}$. Recalling that $d_{\text {match }}$ is positive-definite, for every $(\vec{u}, \vec{v}) \in \Delta^{+}, \beta_{\left(\min _{i} l_{i}\right)^{-1} \varphi_{(\vec{u}, \vec{v})}} \equiv \beta_{\left(\min _{i} l_{i}\right)^{-1} \psi_{(\vec{u}, \vec{v})}}$ if and only

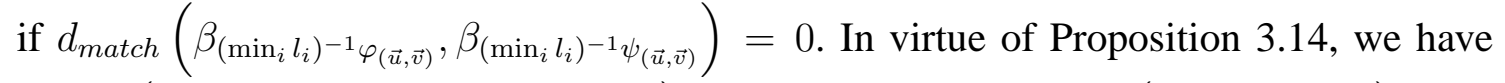
$d_{\text {match }}\left(\beta_{\left(\min _{i} l_{i}\right)^{-1} \varphi_{(\vec{u}, \vec{v})},}, \beta_{\left(\min _{i} l_{i}\right)^{-1} \psi_{(\vec{u}, \vec{v})}}\right)=0$ if and only if $d_{\text {match }}\left(\beta_{\varphi_{(\vec{u}, \vec{v})}, \beta_{\psi_{(\vec{u}, \vec{v})}}}\right)=0$, for every $(\vec{u}, \vec{v}) \in \Delta^{+}$. This proves that $D_{\text {match }}$ is actually positive-definite. The symmetric property is obvious while the triangular inequality follows in a standard way.

Roughly speaking, we have thus proved that small changes in a vector-valued filtering function induce small changes in the associated multidimensional PBNs, with respect to the distance $D_{\text {match }}$.

Our definition of $D_{\text {match }}$ enables us to computationally compare topological data using multidimensional PBNs in the same way as in [16] and, above all, to obtain a lower bound for the natural pseudo-distance as shown in the following section.

\section{The connection between the multidimensional match- ing distance and the natural pseudo-distance}

Another relevant reason to study the multidimensional matching distance $D_{\text {match }}$ is the possibility of obtaining lower bounds for the natural pseudo-distance.

We recall that, for any two topological spaces $X, Y$ endowed with two continuous functions $\vec{\varphi}: X \rightarrow \mathbb{R}^{n}, \vec{\psi}: Y \rightarrow \mathbb{R}^{n}$, we can give the following definition.

Definition 5.1 (Natural pseudo-distance). The natural pseudo-distance between the pairs $(X, \vec{\varphi})$ and $(Y, \vec{\psi})$, denoted by $\delta((X, \vec{\varphi}),(Y, \vec{\psi}))$, is

(i) the number $\inf _{h} \max _{x \in X}\|\vec{\varphi}(x)-\vec{\psi}(h(x))\|_{\infty}$ where $h$ varies in the set $H(X, Y)$ of all the homeomorphisms between $X$ and $Y$, if $X$ and $Y$ are homeomorphic;

(ii) $+\infty$, if $X$ and $Y$ are not homeomorphic.

We point out that the natural pseudo-distance is not a distance because it can vanish on two distinct pairs. However, it is symmetric, satisfies the triangular inequality, and vanishes on two equal pairs. 
The natural pseudo-distance has been studied in $[25,26,27]$ in the case of scalarvalued filtering functions on manifolds, and in [19] in the case of vector-valued filtering functions on manifolds.

As a simple but relevant consequence of the Multidimensional Stability Theorem 4.4 we obtain the following Theorem 5.2, stating that the multidimensional matching distance furnishes a lower bound for the natural pseudo-distance.

Theorem 5.2. Let $X, Y$ be two triangulable spaces endowed with two continuous functions $\vec{\varphi}: X \rightarrow \mathbb{R}^{n}, \vec{\psi}: Y \rightarrow \mathbb{R}^{n}$. Then

$$
D_{\text {match }}\left(\beta_{\vec{\varphi}}, \beta_{\vec{\psi}}\right) \leq \delta((X, \vec{\varphi}),(Y, \vec{\psi})) .
$$

Proof. We follow the same proof line used in [16] for 0th homology. If $H(X, Y)$ is empty our statement is trivially true. Let us assume $H(X, Y) \neq \emptyset$ and take any homeomorphism $h \in H(X, Y)$. We observe that $\beta_{\vec{\psi}}=\beta_{\vec{\psi} \circ h}$. Moreover, for each homeomorphism $h$, by applying the Multidimensional Stability Theorem 4.4, we have

$$
D_{\text {match }}\left(\beta_{\vec{\varphi}}, \beta_{\vec{\psi}}\right)=D_{\text {match }}\left(\beta_{\vec{\varphi}}, \beta_{\vec{\psi} \circ h}\right) \leq \max _{x \in X}\|\vec{\varphi}(x)-\vec{\psi}(h(x))\|_{\infty}
$$

Since this is true for any homeomorphism $h$ between $X$ and $Y$, it immediately follows that $D_{\text {match }}\left(\beta_{\vec{\varphi}}, \beta_{\vec{\psi}}\right) \leq \delta((X, \vec{\varphi}),(Y, \vec{\psi}))$.

We point out that, taking the maximum over all homology degrees, Theorem 5.2 yields a lower bound that improves the one given in [16].

We recall that the natural pseudo-distance, involving all possible homeomorphisms between two triangulable spaces, is quite difficult to compute. Theorem 5.2 could represent a useful and simple tool to estimate this metric.

Acknowledgments. The authors thank Francesca Cagliari (University of Bologna) and Marco Grandis (University of Genoa) for their helpful advice. However, the authors are solely responsible for any errors.

At the time of submission, the first author was visiting the Pattern Recognition and Image Processing Group, Institute of Computer Graphics and Algorithms, Faculty of Informatics, Vienna University of Technology, Austria, supported by the Austrian Science Fund (FWF) grant no. P20134-N13.

The last author partially carried out this work within the activity of ARCES “E. De Castro", University of Bologna.

\section{A Appendix}

The next example shows that the PBNs function is not right-continuous in the variable $v$ when singular or simplicial homologies are considered instead of Čech homology. We recall that a case concerning the right-continuity in the variable $u$ has been described in Example 2.6.

Example A.1. Let $S \subset \mathbb{R}^{3}$ be a sphere parameterized by polar coordinates $(\theta, \phi),-\frac{\pi}{2} \leq \theta \leq \frac{\pi}{2}$ and $\phi \in[0,2 \pi)$. For every $\phi \in[0,2 \pi)$, consider on $S$ the paths $\gamma_{\phi}^{1}:\left(-\frac{\pi}{2}, 0\right) \rightarrow S$ and $\gamma_{\phi}^{2}:\left(0, \frac{\pi}{2}\right) \rightarrow S$ defined by setting, for $i=1,2, \gamma_{\phi}^{i}(\theta)=\left(\theta^{\prime}, \phi^{\prime}\right)$ with $\theta^{\prime}=\theta$ and $\phi^{\prime}=(\phi+\cot \theta) \bmod 2 \pi$. We observe that each point of the set $S^{*}=\left\{(\theta, \phi) \in S: \theta \neq 0 \wedge|\theta| \neq \frac{\pi}{2}\right\}$ belongs to the image of one and only one path $\gamma_{\phi}^{i}$. 


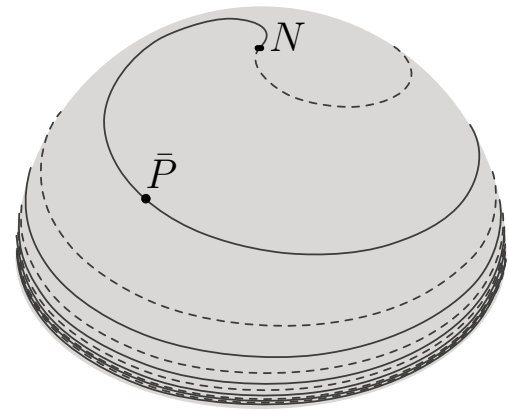

(a)

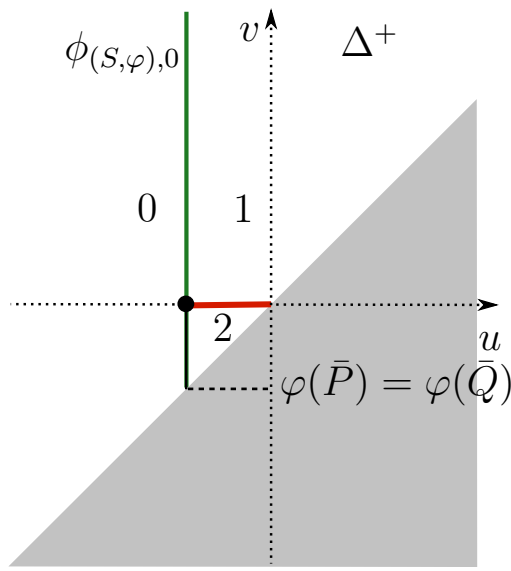

$(b)$

Figure 4: $(a)$ Two of the paths covering the northern hemisphere considered in Example A.1. $(b)$ The 0th PBNs of the function $\varphi$. On the discontinuity points highlighted in bold red, the 0th PBNs computed using singular homology takes a value equal to 2 , while using Čech homology, the value is equal to 1 , showing the right-continuity in the variable $v$.

Such curves approach more and more a pole of the sphere on one side and the equator, winding an infinite number of times, on the other side (see, for instance, in Figure $4(a)$, the paths $\gamma_{\frac{\pi}{2}}^{2}$ and $\gamma_{\frac{3 \pi}{2}}^{2}$ lying in the northern hemisphere).

Then define the $C^{\infty}$ function $\varphi^{*}: S^{*} \rightarrow \mathbb{R}$ that takes each point $P=\gamma_{\phi}^{i}(\theta) \in S^{*}$ to the value $\exp \left(-\frac{1}{\theta^{2}\left(\frac{\pi}{2}-|\theta|\right)^{2}}\right) \sin (\phi)$. Now extend $\varphi^{*}$ to a $C^{\infty}$ function $\varphi: S \rightarrow \mathbb{R}$ in the only way possible. In plain words, this function draws a ridge for $\phi \in(0, \pi)$, and a valley for $\phi \in(\pi, 2 \pi)$. Moreover, observe that the points $\bar{P} \equiv\left(\frac{\pi}{4}, \frac{3 \pi}{2}\right)$ and $\bar{Q} \equiv\left(-\frac{\pi}{4}, \frac{3 \pi}{2}\right)$ of the sphere are the unique local minimum points of $\varphi$.

Let us now consider the 0th PBNs of $\varphi$. Its graph is depicted in Figure $4(b)$. The points $\bar{P}$ and $\bar{Q}$ belong to the same arcwise connected component of the lower level set $S\langle\varphi \leq \varepsilon\rangle$ for every $\varepsilon>0$, whereas they do not for $\varepsilon=0$, since the paths $\gamma_{\frac{\pi}{2}}^{i}(i=1,2)$ are an "obstruction" to constructing a continuous path from $\bar{P}$ to $\bar{Q}$. Hence, the singular PBNs $\beta_{\varphi}$ for 0th homology is not right-continuous in the second variable at $v=0$, for any $u$ with $\min \varphi \leq u<0$.

\section{References}

[1] Biasotti, S., De Floriani, L., Falcidieno, B., Frosini, P., Giorgi, D., Landi, C., Papaleo, L., and Spagnuolo, M. ACM Comput. Surv. 40(4), 1-87 (2008).

[2] Carlsson, G. Bull. Amer. Math. Soc. 46(2), 255-308 (2009).

[3] Carlsson, G., Zomorodian, A., Collins, A., and Guibas, L. J. International Journal of Shape Modeling 11(2), 149-187 (2005).

[4] Cerri, A., Ferri, M., and Giorgi, D. Graph. Models 68(5), 451-471 (2006).

[5] Moroni, D., Salvetti, M., and Salvetti, O. In MDA '08: Proceedings of the 3rd international conference on Advances in Mass Data Analysis of Images and Signals in 
Medicine, Biotechnology, Chemistry and Food Industry, 123-138 (Springer-Verlag, Berlin, Heidelberg, 2008).

[6] Verri, A., Uras, C., Frosini, P., and Ferri, M. Biol. Cybern. 70, 99-107 (1993).

[7] Edelsbrunner, H., Letscher, D., and Zomorodian, A. Discrete \& Computational Geometry 28(4), 511-533 (2002).

[8] de Silva, V. and Ghrist, R. Algebr. Geom. Topol. 7, 339-358 (2007).

[9] Edelsbrunner, H. and Harer, J. In Surveys on discrete and computational geometry, volume 453 of Contemp. Math., 257-282. Amer. Math. Soc., Providence, RI (2008).

[10] Edelsbrunner, H. and Harer, J. Computational Topology: An Introduction. American Mathematical Society, (2009).

[11] Ghrist, R. Bull. Amer. Math. Soc. (N.S.) 45(1), 61-75 (electronic) (2008).

[12] Zomorodian, A. J. Topology for computing, volume 16 of Cambridge Monographs on Applied and Computational Mathematics. Cambridge University Press, Cambridge, (2005).

[13] Carlsson, G. and Zomorodian, A. Discrete \& Computational Geometry 42(1), 71-93 (2009).

[14] Frosini, P. and Landi, C. Pattern Recognition and Image Analysis 9, 596-603 (1999).

[15] Kaczynski, T., Mischaikow, K., and Mrozek, M. Computational Homology. Number 157 in Applied Mathematical Sciences. Springer-Verlag, 1 edition, (2004).

[16] Biasotti, S., Cerri, A., Frosini, P., Giorgi, D., and Landi, C. J. Math. Imaging Vision 32(2), 161-179 (2008).

[17] Cagliari, F., Di Fabio, B., and Ferri, M. Proc. Amer. Math. Soc. 138(8), 3003-3017 (2010).

[18] Carlsson, G., Singh, G., and Zomorodian, A. In ISAAC '09: Proceedings of the 20th International Symposium on Algorithms and Computation, 730-739 (SpringerVerlag, Berlin, Heidelberg, 2009).

[19] Frosini, P. and Mulazzani, M. Bulletin of the Belgian Mathematical Society 6(3), 455-464 (1999).

[20] Cohen-Steiner, D., Edelsbrunner, H., and Harer, J. Discrete Comput. Geom. 37(1), 103-120 (2007). 
[21] Cohen-Steiner, D., Edelsbrunner, H., Harer, J., and Mileyko, Y. Foundations of Computational Mathematics 10(2), 127-139 (2010).

[22] Chazal, F., Cohen-Steiner, D., Glisse, M., Guibas, L. J., and Oudot, S. Y. In SCG '09: Proceedings of the 25th annual symposium on Computational geometry, 237246 (ACM, New York, NY, USA, 2009).

[23] d'Amico, M., Frosini, P., and Landi, C. Acta. Appl. Math. 109, 527-554 (2010).

[24] Carlsson, G. and Zomorodian, A. In SCG '07: Proceedings of the twenty-third annual symposium on Computational geometry, 184-193 (ACM, New York, NY, USA, 2007).

[25] Donatini, P. and Frosini, P. Forum Mathematicum 16(5), 695-715 (2004).

[26] Donatini, P. and Frosini, P. Journal of the European Mathematical Society 9(2), 231253 (2007).

[27] Donatini, P. and Frosini, P. Forum Mathematicum 21(6), 981999 (2009).

[28] Robins, V. Topology Proceedings 24(1), 503-532 (1999).

[29] Robins, V. Computational topology at multiple resolutions. $\mathrm{PhD}$ thesis, University of Colorado, (2000).

[30] Mrozek, M. Discrete and Computational Geometry 44, 546-576 (2010).

[31] Eilenberg, S. and Steenrod, N. Foundations of algebraic topology. Princeton University Press, Princeton, New Jersey, (1952).

[32] Rourke, C. P. and Sanderson, B. J. Introduction to piecewise-linear topology. Springer-Verlag, New York, (1972). Ergebnisse der Mathematik und ihrer Grenzgebiete, Band 69.

[33] Frosini, P. and Landi, C. Appl. Algebra Engrg. Comm. Comput. 12(4), 327-349 (2001).

[34] Greenberg, M. J. and Harper, J. R. Algebraic topology: a first course. Number 58. Addison-Wesley Publishing Company, (1981).

[35] Cerri, A. and Frosini, P. Technical Report no. 2765, Università di Bologna, (2010). Available at http://amsacta.cib.unibo.it/2765/. 